\title{
CERTAIN WEIGHTED MEAN INEQUALITY
}

\author{
NAMKWON KIM \\ Department of Mathematics, Chosun University, KWAngJu 501-759, Korea \\ E-mail address: kimnamkw@chosun.ac.kr
}

ABSTRACT. In this paper, we report a new sharp inequality of interpolation type in $\mathbb{R}^{n}$. This inequality is for controlling weighted average of a function via $L^{n}$ norm of the gradient of a function together with its' certain exponential norm.

\section{INTRODUCTION}

Inequalities of interpolation type are well-known nowadays and very important in studying PDEs(See for instance [1,3] and references therein). These inequalities bound roughly $L^{p}$ norm of a function itself by the derivatives of the function. Concretely, for a function $u \in$ $W^{1, r}\left(\mathbb{R}^{n}\right)$, we have

$$
\|u\|_{L^{p}} \leq C\|\nabla u\|_{L^{r}}^{t}\|u\|_{L^{r}}^{1-t}, \quad r \leq p \leq p^{*} \equiv \frac{n r}{n-r} .
$$

Here, $t \in[0,1]$ is determined by $p, r$. But, this type of Sobolev inequality fails when $p<r$. In fact, for a cutoff function $\xi$ and large $R>0$, if we take

$$
u=C \xi\left(\frac{|x|}{R}\right), \quad C>0
$$

then $\|u\|_{L^{p}} \sim R^{n / p},\|\nabla u\|_{L^{r}} \sim R^{-1+n / r},\|u\|_{L^{r}} \sim R^{n / r}$ and (1.1) fails as $R \rightarrow \infty$. However, if we consider $u$ which is average zero even under a weighted sense, such inequality remains true in many cases by the Poincare inequality[2]. Thus, it is crucial to bound a weighted average of a function if we want to have inequalities like (1.1). As far as the author knows, an inequality with such spirit appears in [6] firstly and turns out to be helpful to problems in gauge theory. The purpose of this paper is to report such inequality using certain exponential integral of a function. That is,

$$
\left|\int_{\mathbb{R}^{n}} g v\right| \leq \frac{1}{n}\left(\frac{\omega_{n}}{n}\right)^{(n-1) / n}\|\nabla v\|_{L^{n}\left(\mathbb{R}^{n}\right)}[\ln (X+1)+C]^{\frac{n-1}{n}}+C
$$

for any $v \in W^{1, n}$. Here, $g=\left(1+|x|^{n}\right)^{-2}, X=\int_{\mathbb{R}^{n}}\left(e^{-|v|}-1\right)^{2}$, and $C$ is an absolute constant depending only on $n$. In view of the already existing Poincare inequality, The above

Received by the editors September 2 2014; Accepted September 5 2014; Published online September 152014. 2000 Mathematics Subject Classification. 26D10, 35J99.

Key words and phrases. weighted mean inequality, interpolation inequality.

This work is partially supported by Chosun university, 2008. 
guarantees that $L_{l o c}^{p}, p \leq \frac{n p}{n-p}$ norm of $v$ can be bounded by the RHS of (1.2). We also show that (1.2) is sharp providing an example which do not satisfy (1.2) if we replace the coefficient $\frac{1}{n}\left(\frac{\omega_{n}}{n}\right)^{(n-1) / n}$ with any smaller constant. We finally remark that exponential integral in the righthand side of (1.2) can be replaced with integral of similar bounded function. In fact, if we replace $\left(e^{-|v|}-1\right)^{2}$ with $\phi^{2}(v)$ satisfying

$$
\phi(t) \geq C \min \{1, t\}, \text { for } t>0,
$$

(1.2) holds true due to

$$
\int\left(e^{-|v|}-1\right)^{2} \leq \sup _{t>0} \frac{\left(e^{-t}-1\right)^{2}}{\phi^{2}(t)} \int \phi^{2}(v) \leq C \int \phi^{2}(v) .
$$

\section{WEIGHTED MEAN INEQUALITY}

From now on, we omit the domain of integration if it is $\mathbb{R}^{n}$. We also denote $|x|=r$ and $g=\left(1+r^{n}\right)^{-2}$ as before. We first remind that symmetrization holds true for any nonnegative smooth function with compact support.

Theorem 2.1. There exists $C>0$ such that

$$
\left|\int g v\right| \leq \frac{1}{n}\left(\frac{\omega_{n}}{n}\right)^{(n-1) / n}\|\nabla v\|_{L^{n}}[\ln (X+1)+C]^{(n-1) / n}+C
$$

for $v \in W^{1, n}$. Here, $X$ is as before and $\omega_{n}$ is the volume of the $n-1$ dimensional unit sphere.

Proof) It is clear that

$$
\left|\int g v\right| \leq\left|\int g \times-\right| v||, \quad\|\nabla|v|\|_{L^{n}} \leq\|\nabla v\|_{L^{n}}
$$

Thus, it is enough to show (1.2) replacing $v$ with $-|v|$. Since $-|v|$ itself belongs to $W^{1, n}$, we can assume $v \leq 0$ without loss of generality. Then, it is again enough to show (1.2) for $v \in C_{0}^{\infty}$ with $v \leq 0$ by the standard density theorem. If $v$ is nonpositive and of compact support, we can apply the symmetrization on $-v$. Let us denote the nonincreasing rearrangement of $-v$ by $(-v)_{S}$. Clearly, $v^{*} \equiv-(-v)_{S}$ is nondecreasing with respect to $r$. Further, $g$ is decreasing with respect to $r$ and, due to $v \leq 0,1-e^{v}$ and $1-e^{v^{*}}$ are equi-measurable. Therefore,

$$
\int g v \geq \int g v^{*}, \quad \int\left(e^{v}-1\right)^{2}=\int\left(e^{v^{*}}-1\right)^{2}, \quad\left\|\nabla v^{*}\right\|_{L^{r}} \leq\|\nabla v\|_{L^{r}} .
$$

Thus, it is enough to show (1.2) for radially symmetric nonpositive smooth $v$ with compact support.

Now, for $R>0$,

$$
v(r)=v(R)+\int_{R}^{r} \partial_{s} v(s) d s .
$$

We multiply the above by $g(r) r^{n-1}$ and integrate with respect to $r$ on $(0, \infty)$ to get

$$
L H S=\int_{0}^{\infty} v(r) g(r) r^{n-1} d r
$$




$$
\begin{aligned}
R H S & =C v(R)+\int_{0}^{\infty} g(r) r^{n-1} \int_{R}^{r} \partial_{s} v(s) d s d r \\
& =C v(R)+\left(\int_{0}^{R}+\int_{R}^{\infty}\right) g(r) r^{n-1} \int_{R}^{r} \partial_{s} v(s) d s d r=C v(R)+I+I I .
\end{aligned}
$$

By the Fubini theorem,

$$
\begin{aligned}
|I| & \leq \int_{0}^{R}|\nabla v(s)| \int_{0}^{s} g(r) r^{n-1} d r d s=\int_{0}^{R}|\nabla v(s)| \frac{s^{n}}{n\left(1+s^{n}\right)} d s \\
& \leq \frac{1}{n} \omega_{n}^{-\frac{1}{n}}\|\nabla v\|_{L^{n}}\left(\int_{0}^{R}\left(\frac{s^{n-(n-1) / n}}{1+s^{n}}\right)^{\frac{n}{n-1}} d s\right)^{(n-1) / n}, \\
|I I| & \leq \int_{R}^{\infty}\left|\partial_{s} v(s)\right| \int_{s}^{\infty} g(r) r^{n-1} d r d s=\int_{R}^{\infty}\left|\partial_{s} v(s)\right| \frac{1}{n\left(1+s^{n}\right)} d s \\
& \leq \frac{1}{n} \omega_{n}^{-\frac{1}{n}}\|\nabla v\|_{L^{n}}\left(\int_{R}^{\infty} \frac{1}{s}\left(1+s^{n}\right)^{-\frac{n}{n-1}} d s\right)^{(n-1) / n}
\end{aligned}
$$

Here, $\omega_{n}$ is the volume of $S^{n-1}$. By change of variables $s^{n}=t$, when $R>1$,

$$
\begin{aligned}
\int_{0}^{R}\left(\frac{s^{n-(n-1) / n}}{1+s^{n}}\right)^{\frac{n}{n-1}} d s & =\frac{1}{n} \int_{0}^{R^{n}}\left(\frac{1}{1+t}\right)^{\frac{n}{n-1}} t^{\frac{1}{n-1}} d t \\
& \leq \frac{1}{n} \int_{0}^{R^{n}} \frac{1}{1+t}=\frac{1}{n} \ln \left(1+R^{n}\right) \\
\int_{R}^{\infty} \frac{1}{s}\left(1+s^{n}\right)^{-\frac{n}{n-1}} d s & \leq \frac{1}{n} \int_{1}^{\infty} \frac{1}{t}(1+t)^{-\frac{n}{n-1}} d t \leq C .
\end{aligned}
$$

Thus, we get

$$
|I|+|I I| \leq n^{-\frac{2 n-1}{n}} \omega_{n}^{-1 / n}\|\nabla v\|_{L^{n}}\left(\ln \left(C+R^{n}\right)\right)^{(n-1) / n} .
$$

Meanwhile, we claim that for some $C>0$, there exists $R_{1} \leq C X^{1 / n}$ such that $v\left(R_{1}\right)>-2$. Indeed, otherwise, we would have $v(r)<-2$ uniformly on the interval $T \equiv\left[0, C X^{1 / n}\right]$. Then,

$$
X=\int\left(e^{v}-1\right)^{2} d x \geq \frac{\omega_{n}}{4} \int_{T} r^{n-1} d r=\frac{\omega_{n}}{4 n} C^{n} X
$$

This gives a contradiction if we choose $C>\left(4 n / \omega_{n}\right)^{1 / n}$. Thus, the claim is proved. We take $R=R_{1}$ in (2.2) and arrive at

$$
\left|\int g v\right|=\omega_{n}\left|\int_{0}^{\infty} g v r^{n-1} d r\right| \leq C+\frac{1}{n}\left(\frac{\omega_{n}}{n}\right)^{(n-1) / n}\|\nabla v\|_{L^{n}}\left(\left(\ln \left(C+R_{1}^{n}\right)\right)^{\frac{n-1}{n}}+C\right) .
$$

Since $R_{1} \leq C X^{1 / n}$, we arrive at the desired result redefining $C$ suitably.

Now, we give an example which shows the sharpness of (1.2). Consider the functions, 


$$
\psi(x)= \begin{cases}-\ln R & |x| \leq 1 \\ \ln \left(\frac{r}{R}\right) & 1<|x|<R \\ 0 & |x| \geq R\end{cases}
$$

It is clear that

$$
\begin{gathered}
\|\nabla \psi\|_{L^{n}}=\left(\omega_{n} \int_{1}^{R} \frac{1}{r^{n}} r^{n-1} d r\right)^{1 . n}=\left(\omega_{n} \ln R\right)^{1 / n}, \\
X \sim C+C \int_{1}^{R}\left(1-\frac{r}{R}\right)^{2} r^{n-1} d r \sim C+C R^{n}, \\
\int g \psi=\omega_{n} \int_{0}^{\infty} \frac{-\ln R}{\left(1+r^{n}\right)^{2}} r^{n-1} d r+C=C-\frac{\omega_{n}}{n} \ln R .
\end{gathered}
$$

Thus, (1.2) fails for $\psi$ as $R \rightarrow \infty$ if we replace $\frac{1}{n}\left(\frac{\omega_{n}}{n}\right)^{(n-1) / n}$ in (1.2) with smaller coefficient.

\section{ACKNOWLEDGEMENT}

This work was partially supported by Chosun university, 2008.

\section{REFERENCES}

[1] S. Agmon, A. Douglis, and L. Nirenberg, Estimates near the boundary for solutions of elliptic partial differential equations satisfying general boundary conditions I, Comm. Pure Appl. Math. 12(1959), 623-727.

[2] L. C. Evans and R. F. Gariepy, "Measure theory and fine properties of functions", CRC press, Florida, FL, 1992.

[3] L. Nirenberg, On elliptic partial differential equations, Ann. Scuola Norm. Sup. Pisa (3) 13(1959), $115-162$.

[4] G. Talenti, Best constant in Sobolev inequality, Annali di Mat. pura ed Applicata 110(1976), 353-372.

[5] N.S. Trudinger, On imbeddings into Orlicz. Sobolev spaces and some applications, J. Math. Mech. 17(1967), 473-483.

[6] R. Wang, Chern-Simons vortices, Comm. Math. Phys. 137(1991), 587-597. 